\title{
Structural Systems Composed of Concentric Hoops and Designed for Lightweight Domes of Large Spans
}

\author{
Janusz Rebielak \\ Faculty of Architecture, Cracow University of Technology, Kraków 31-155, Poland
}

\begin{abstract}
The paper presents a selected group of tension-strut structural systems designed for the construction of lightweight dome covers of large spans, which can be comparatively easy to assembly and have rises of which can be relatively small. This will allow significant decrease costs of erection and maintenance of objects covered by these roof structures. The proposed systems have been obtained from the results of suitable transformations of a chosen type of double-layer space frame and an appropriate arrangement of tetrahedron modules in the space of each of the newly designed type of the structural system. All these systems are built by means of concentric hoops having their own integral spatial stiffness obtained after an appropriate pre-stressing. Particular hoops can be mounted on the ground level and then one by one will be hoisted to the designed positions where they will be connected by means of special sets of the tension members. Due to these structural features, the assembly process of each system should be relatively simple, fast and not expensive. The whole tension-strut structure has to be connected to the compression perimeter ring and suitably pre-stressed. There are presented visualizations of the proposed systems prepared on the basis of the appropriate numerical models especially defined for each particular structure.
\end{abstract}

Key words: Roof cover, dome structure, structural system, pre-stressing, tension-strut structure.

\section{Introduction}

A spherical surface has some specific geometric characteristics related to the double curvatures of its surface which make the problem of the design and construction of a large span roof structure by application of straight members a very difficult task.

The issue of design of reliable and economic dome structures is constantly a significant challenge for architects and engineers. In the past, the domes were erected by means of traditional materials like timber, stone or bricks. At the beginning of 19th century, iron and later steel were widely applied in roof structures including domes, which made them lighter and able to cover larger areas. Structural members transmitting tension forces are mostly essentially lighter than members, which transmit compression forces of equal

Corresponding author: Janusz Rebielak, Ph.D., professor, research fields: architecture, numerical methods of shaping and design of the space structures, methods of static calculations, lightweight structures for large span roofs, tall buildings and foundation systems. E-mail: j.rebielak@wp.pl. values what in a simple way is presented in works [1-3]. Because the dead load of a large span roof structure is proportional, in a certain relationship, to the square of its clear span that is why application of a system, which consists of possibly a small number of compression members and the necessary number of tension members, is considered as the advantageous technical solution what is discussed in Refs. [4-6]. Although the air-supported structures theoretically can be of an unlimited clear span, their practical applications are restricted by numerous architectonic, useful, safety or economic reasons [7, 8]. Many technical and useful reasons have inclined architects and engineers to search for new structural concepts of the dome covers. Tension-strut systems belong nowadays to group of the most effective structural solutions for roofs of the large spans [9]. This paper presents a review of selected types of structural systems developed recently by the author and proposed for the lightweight dome covers of large spans. 


\section{Basic Form of the Proposed Space Structural Systems}

Space structures in form of spatial trusses were considered in the middle of the last century as the very lightweight support structures for various types of the roof covers [10]. Necessity of the exact statically calculations and structural analyses of these multifold statically indeterminate systems has resulted in significant development of new methods of structural design and other techniques or technologies used in the civil engineering and what is presented in works $[11,12]$. The arrangement of the component parts inside the crystal space frame (Fig. 1) was taken as the basic structural configuration for the transformations conducted to achieve a double-layer ring-net dome of unique architectonic form and for advantageous conditions for lighting the inner space by the diffused daylight.

For instance, panels of the upper layer, represented in Fig. 1 by triangular panel ABC, can be typical opaque components of the roof cladding, while panels of the type ACD are proposed to be the translucent window panels, which is described in Ref. [13]. Other groups of opaque triangular panels should be arranged between skew members of the upper layer, located, e.g., between nodes A and B or between nodes A and $\mathrm{C}$, and the cross members in the way is shown in Fig. 1.

The inner build of the above defined structure has been somewhat converted and adjusted to the needs of the double-layer ring-net dome, schemes of which are presented in Fig. 2. This form of a spherical system is proposed to call the sunflower dome structure.

It is characterized by several interesting morphological features, that is why this structural configuration was subjected to the further transformations carried out in order to obtain other types of very efficient and also lightweight dome systems.

\section{Crystal Tension-Strut Structure}

Domes constructed by means of traditional or considered as conventional structural systems have to have a rather big rise of the cover, compared with Fig. 2 , which is conditioned by the smallest ratio of rise and clear span of the cover. This implies that volume of the inner air, the temperature of which has to be controlled, increases rapidly when the clear span of the cover ascends. In order to decrease the maintenance costs of such an object, the dome cover should be of the smallest possible rise. Large span domes of very small rise can be successfully realized by the application of unique types of tension-strut structural systems. The most well-known is the cable dome system invented by David Geiger and firstly

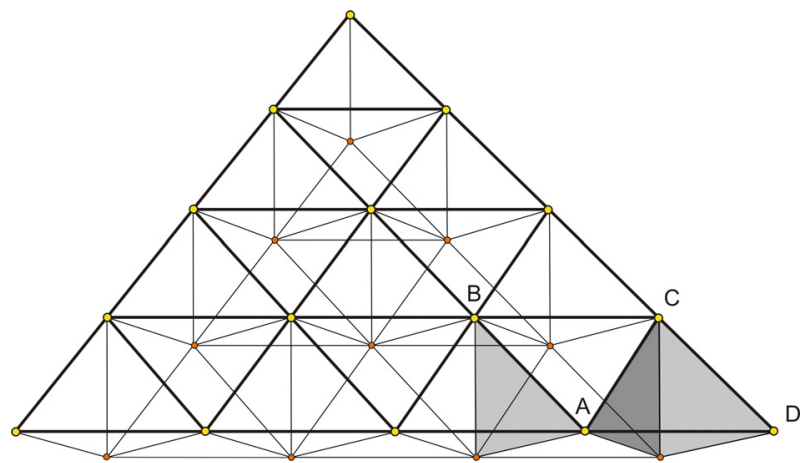

Fig. 1 Part of the crystal space frame with triangular panels arranged between selected structural members.

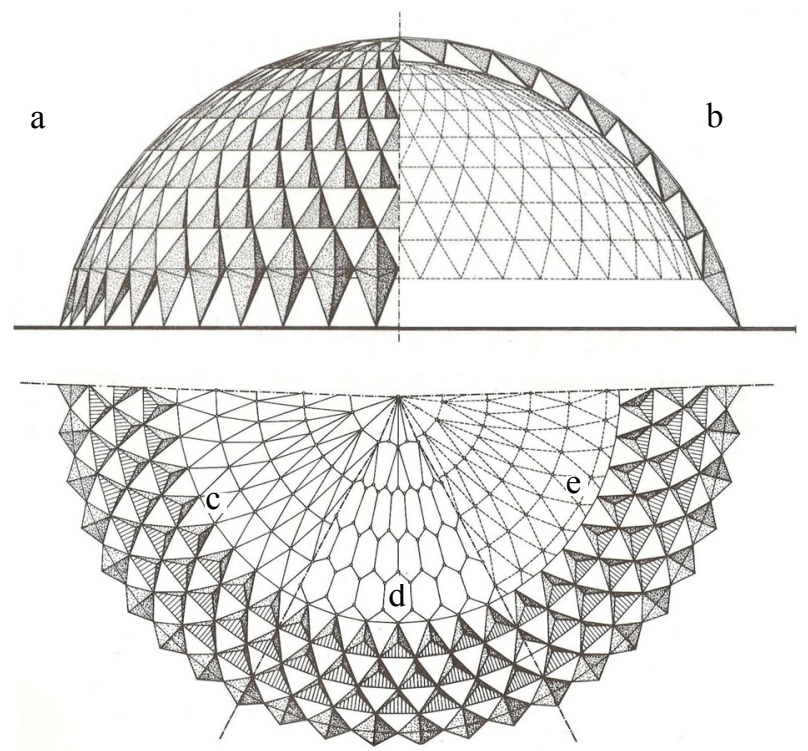

Fig. 2 (a) Elevation of specific spherical form of the crystal space structure called the sunflower dome structure; (b) vertical cross-section; (c) arrangement of members of the upper layer; (d) spacing of the cross-braces; (e) arrangement of members of the lower layer. 
built for Olympic Games in Korea in 1988 [14, 15]. It is worthy to mention that a very similar structural concept was invented earlier by Wacław Zalewski and applied in the roof structure of the Sport-Convention Hall "Spodek" in Katowice built at the end of the 1960s, the beginning of the 1970s in Poland [16]. The structural concept of the cable dome system is totally different from the concepts of common types of the lattice dome structures. Because of the necessity of pre-stressing, it may be considered as a specific type of the tensegrity system, earlier defined by Kenneth Snelson and Buckminster Fuller. The assembly process of the Geiger's cable dome structure has to be done very precisely, it is complex and the completed structure may be sensitive to non-uniformly applied load.

The first system presented is an outcome of the aim for obtaining a tension-strut structure that is more stable, which moreover could be easier to assemble than the cable dome structure. It was shaped as a result a transformation of the inner build of the crystal space frame, compared with Fig. 1, being the bearing structure of the double-layer lattice dome, compared with Fig. 2, supported in a perimeter ring. The transformation was done in two stages. In the first stage, the statuses of the most members were changed to tension members. Only the cross braces (St in Fig. 3) remained as the struts (Fig. 3). In the second stage, all of the skew members are removed from the lower layer which implies that the only members remaining are running along the concentric hoops ( $\mathrm{H}$ in Fig. 3). A system shaped in this way is called the crystal tension-strut structure, which has to be suitably pre-stressed and connected to the perimeter compression ring ( $\mathrm{R}$ in Fig. 3) what is described in Ref. [17]. The pre-stressing can be introduced in several various ways, for instance, by making shorter the segments of concentric hoops ( $\mathrm{H}$ in Fig. 3) or by reducing the length of diagonal cables ( $\mathrm{E}$ in Fig. 3 ). The sloping of these diagonal cables should not be too small, its boundary value is estimated as $15^{\circ}$, which is dictated by the tendency to decrease values of necessary pre-stressing, due to which values of forces acting in the members of this structure could be relatively small [18].

The roof cladding is designed in form of membrane sheets arranged in triangular fields between cables of the upper layer (Uc and Uh in Fig. 3). This triangular cable network in the upper layer makes the whole structural system very stable. The crystal tension-strut structure is composed of a couple of concentric tension-strut hoops, which can be relatively easily mounted at ground level without the necessity of using the complex hoisting equipment. Then these hoops can be successively lifted up to the designed positions and connected to the other members of this system, which should make the assembly process easier, faster and probably cheaper than the same process of typical cable domes structures.

Some structural features justify the expectation that the system could be a very effective technical solution for the construction of large span dome covers. In this case, the structure should consist of several concentric hoops, construction depths of which have to be appropriately defined. Hoops of the biggest construction depth have to be situated close to the
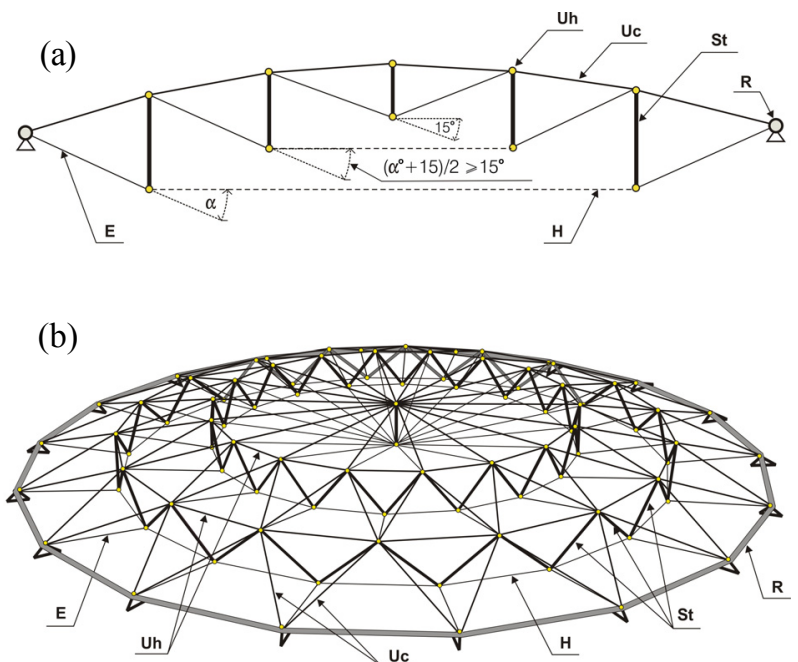

Fig. 3 General schemes of structural system obtained as result of suitable transformations of the crystal space frame: (a) scheme of the main vertical cross-section of simple form of the crystal tension-strut structure; (b) general view of the structure. 
perimeter compression ring. System of the already determined shape of the crystal tension-strut structure can be subjected to further transformations. Because the basic type is characterized by large spatial stability, it has been decided to eliminate all the upper tension members (Uh in Fig. 3) from each of the concentric hoops. These cables should be taken away after the assembly process of the basic type of the crystal tension-strut structure. The form of the structure with a reduced number of members in the upper layer is shown in Fig. 4.

The considerably large spatial stability of basic form of the crystal tension-strut structure leads to a conclusion that the system can be adapted for the needs of a large scale dome cover with a huge central opening (Fig. 5). In this case, the upper members of each concentric hoop have to be made as struts, they should be able to take the compression forces acting

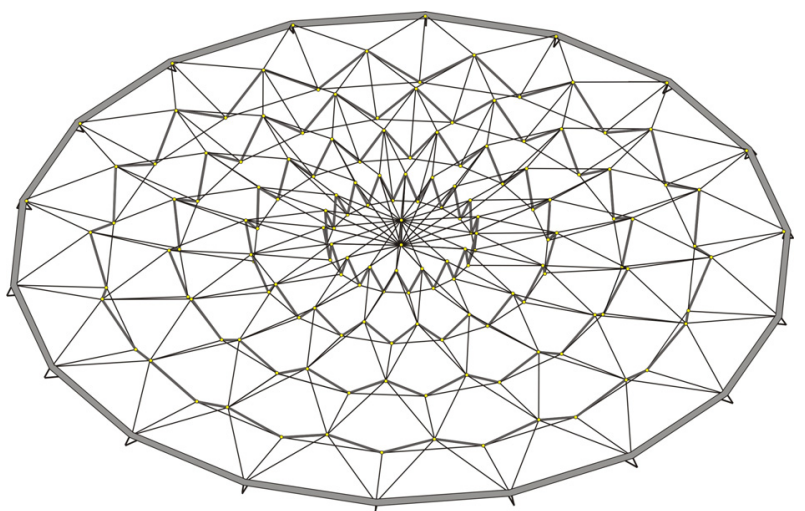

Fig. 4 Shape of the crystal tension-strut structure with reduced number of tension members in the upper layer.

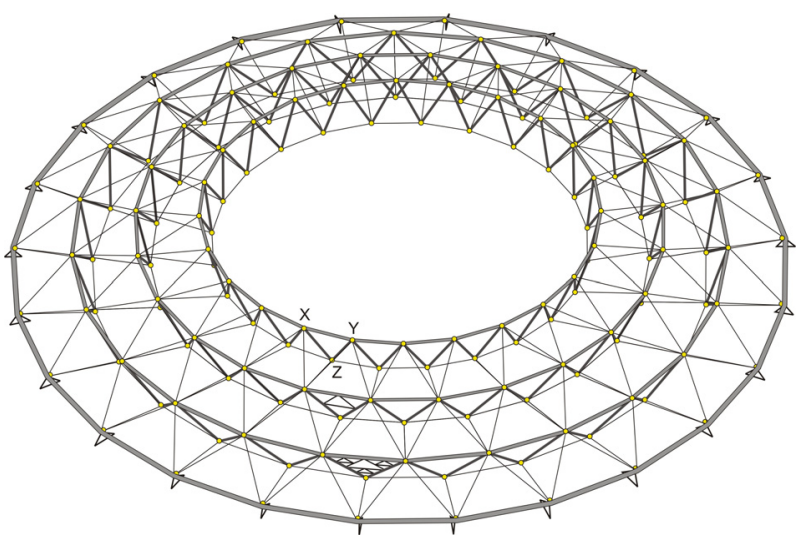

Fig. 5 View of an example form of the crystal tension-strut structure with the central opening. there not only during the assembly process. The positions of these members are indicated by an exemplary segment XY (Fig. 5) being an upper edge of the triangle XYZ (Fig. 5). The lengths of these struts are bigger in successive hoops located closer to the perimeter ring. Therefore, it is recommended to arrange the secondary members inside areas of the upper triangular fields of the hoops. This helps to decrease the buckling lengths of struts, which in an area close to the dome perimeter have to be of a considerably large length.

\section{Structural Systems Shaped by the Application of Some Additional Tetrahedron Modules}

The last presented subtype of the crystal tension-strut structure with a central opening has been assumed as a basis for further transformations. The structure of a very large span consists of the main components, having also huge geometrical dimensions like the particular concentric hoops. They have to be of the suitable spatial stability, which is especially important during their hoisting. This is why it has been decided to increase the spatial stiffness of these hoops by an arrangement of appropriate tetrahedron modules spaced over selected triangular fields. These modules can be situated in various ways, which gives as a result of a numerous group of structural solutions. Diversity of structural shapes possible to obtain in this way was assumed to define by means of relatively simple way of denotation of their types. Struts have to be placed at least at three edges of each module and the axes of these three struts have to be focused in the vertex node of the tetrahedron module. Such modules with V-shaped sets of struts are decided to denote by symbol "V". When the structure is shaped by application of these modules spaced over the upper triangular fields of the basic concentric hoops, then the symbol of a structure begins with the letters "VU". If they are spaced over the lower triangular fields, symbol of the created structure begins with the letters 
"VL". When in space of a structure, the vertex nodes of V-shaped sets of struts are connected together by means of tension members, then its symbol is supplemented by abbreviation "Tens". If they are connected by means of compression members, then in symbol of the created, structure appears abbreviation "Com". Structural systems with central openings have character " $\mathrm{O}$ " in the end of their names. Moreover, other characters and figures can be applied for creating individual denotations for particular types or sub-types of such structural configurations [18].

A new type of structural system is proposed to be built by the means of the tetrahedron modules spaced over every second upper triangular field of each hoop (Fig. 6). Vertex nodes ( $\mathrm{K}$ in Fig. 6) of all these modules are directed towards the perimeter ring $(\mathrm{R}$ in Fig. 6) and they are connected together by means of tension members (P1 in Fig. 6) forming another single concentric hoop. Structural system of this shape, capable of creating a roof structure with a central opening, is denoted the VU-TensO tension-strut structure [18].

When the dimensions of the tetrahedron modules are big, it would be advantageous to apply additional members inside suitable triangular areas, for example, inside the triangle XKY (Fig. 6). The system of the proposed shape is called the VU-TensO and an exemplary form of it, built by means of two main spatial hoops, is presented in Fig. 7. Rules for making specific denotations for these complex systems are presented widely in Ref. [18].

The structure VU-TensO was used as the bearing structure of a huge dome cover called the Hall 2010, presented in Refs. [18, 19]. It was designed as one of the two main objects of the central complex of the World Expo intended to be held in Wroclaw, Poland in 2010 (Figs. 8 and 9).

General message of the planned Expo was the global protection of the natural environment. As it is commonly known, the World Expo 2010 was finally held in Shanghai, China. The Hall 2010 was designed as a multi-purpose dome with a clear span of 365.25 $\mathrm{m}$ (Fig. 8). The span measured in meters is intended as an equivalent of number of days in the year and it exceeds, by about $300 \mathrm{~m}$, the clear span of the Centennial Hall erected in the same city in 1913. The dome is supported by 52 spatial stanchions located at the distance of ca. $22.05 \mathrm{~m}$ along the perimeter compression ring. The number of these supports equals the number of the weeks in the year. The main VU-TensO structure of the roof consists of four concentric spatial hoops (Fig. 9) and this number refers to the number of seasons in the year. The total height of the dome is rather small, about $52.5 \mathrm{~m}$, and it is limited mainly by the functional reasons. The

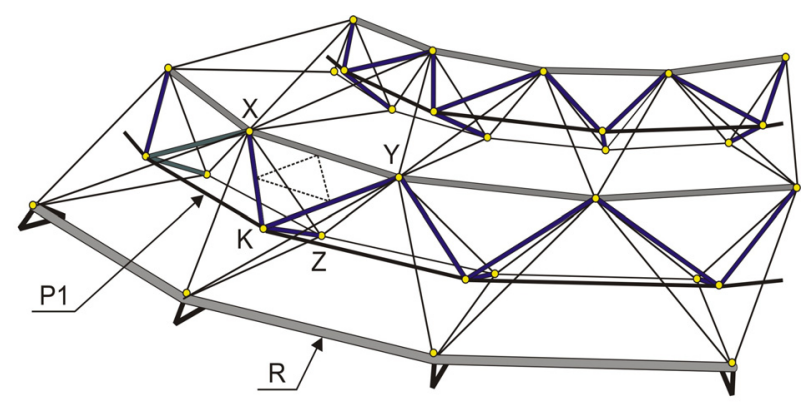

Fig. 6 Scheme of arrangement of component parts in space of the VU-TensO tension-strut structure.
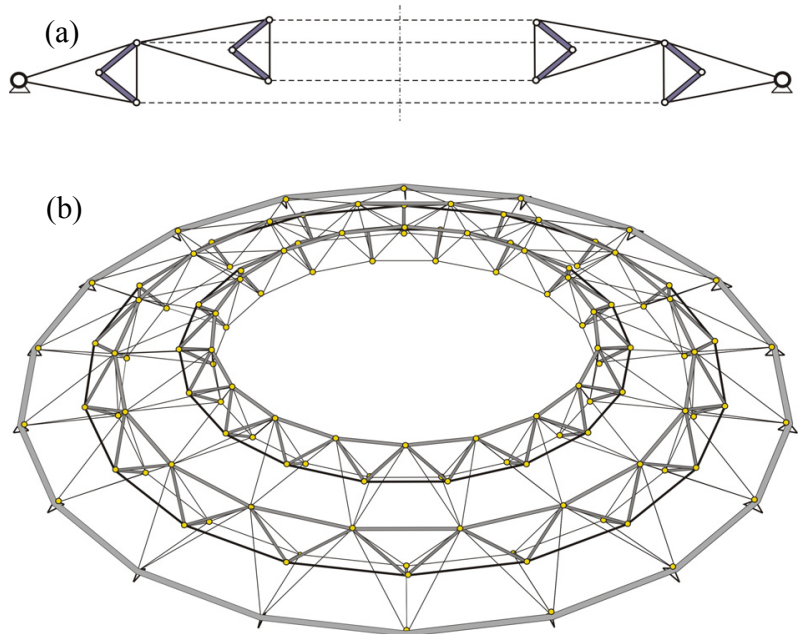

Fig. 7 Inner build of structural system composed of single spatial hoops created by additional tetrahedron modules directed towards the roof perimeter and spaced over upper triangular fields: (a) scheme of the main vertical cross-section of a dome structure; (b) view of the dome structure designed by means of the $\mathrm{VU}$-Tens $\mathrm{O}$ tension-strut structure composed of two concentric spatial hoops. 


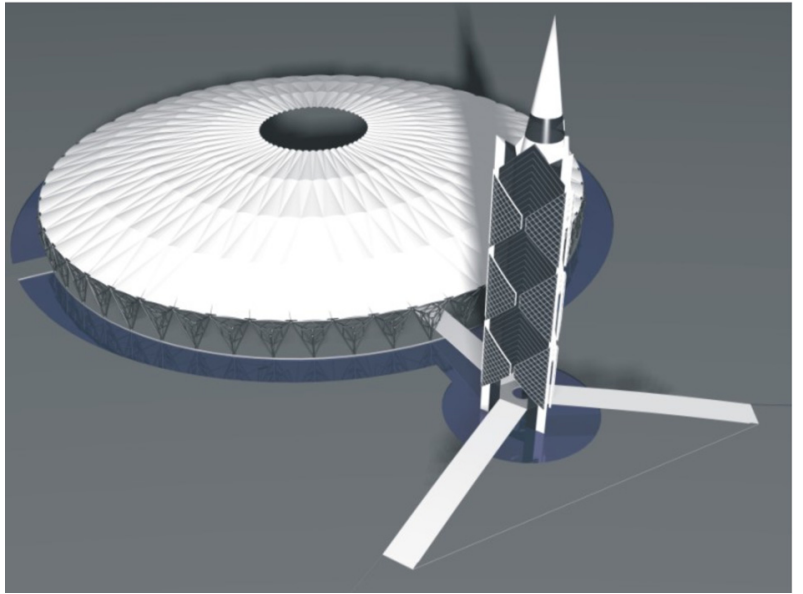

Fig. 8 Overall view of the central complex proposed for the intended Expo 2010 in Wroclaw [19].

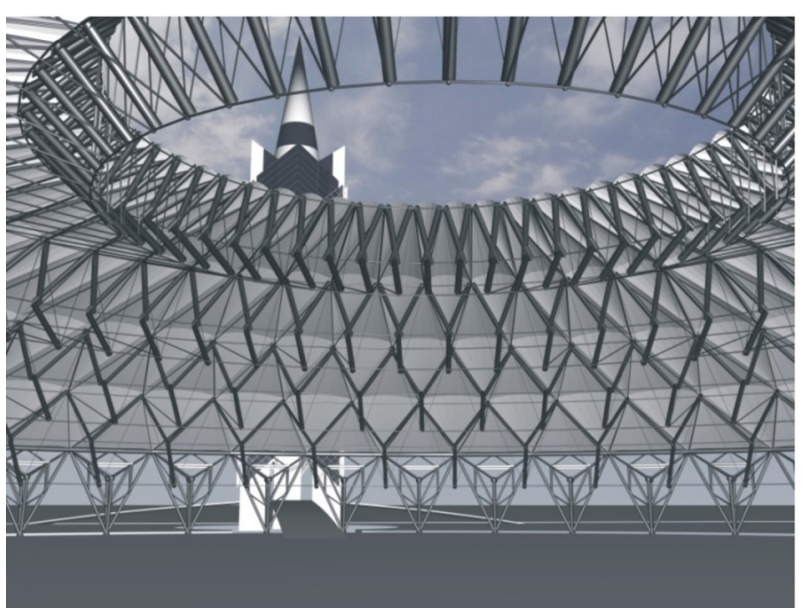

Fig. 9 Perspective view of the interior space of the Hall 2010 [19].

central opening of the clear span equals ca. $73.05 \mathrm{~m}$ and it can be temporary closed by means of a very lightweight and semi-translucent air-inflated structure. The roof cladding system is designed in form of suitably arranged triangular sheets of membranes. A very similar cladding is foreseen for use as a temporary closure of the side perimeter wall of the dome. Advantageous features of the VU-TensO structure justify its application for such a difficult engineering task.

Groups of similar tension-strut structures are numerous because the tetrahedron modules can be arranged in many different ways and moreover, they can be connected together in various manners. The two systems described below can represent the main structural types of these groups. Fig. 10 shows the inner build of a system denoted as the VL-TensZ1dO tension-strut structure and its name is created according to the rules of denotation presented earlier and precisely described in Ref. [18]. It is built of tetrahedron modules with V-shaped sets of struts spaced alternatively over the lower triangular fields of each hoop, for instance, over a triangle ABC (Fig. 10). Vertices of the half of these modules are directed towards the perimeter ring while the second half is directed oppositely, it means towards the cover centre. Adjacent modules in a single hoop are directed alternately and their vertices, e.g., $\mathrm{G}$ and $\mathrm{F}$ (as shown in Fig. 10), are joined by means of tension members (Z1 in Fig. 10) running in a zigzag way into the space of a particular hoop. Spacing of the tetrahedron modules over the lower triangular fields according to the basic structural configuration presented in Ref. [18] may cause some difficulties not only during the assembly of this system. Therefore, it is necessary to apply struts in positions of at least half number of the
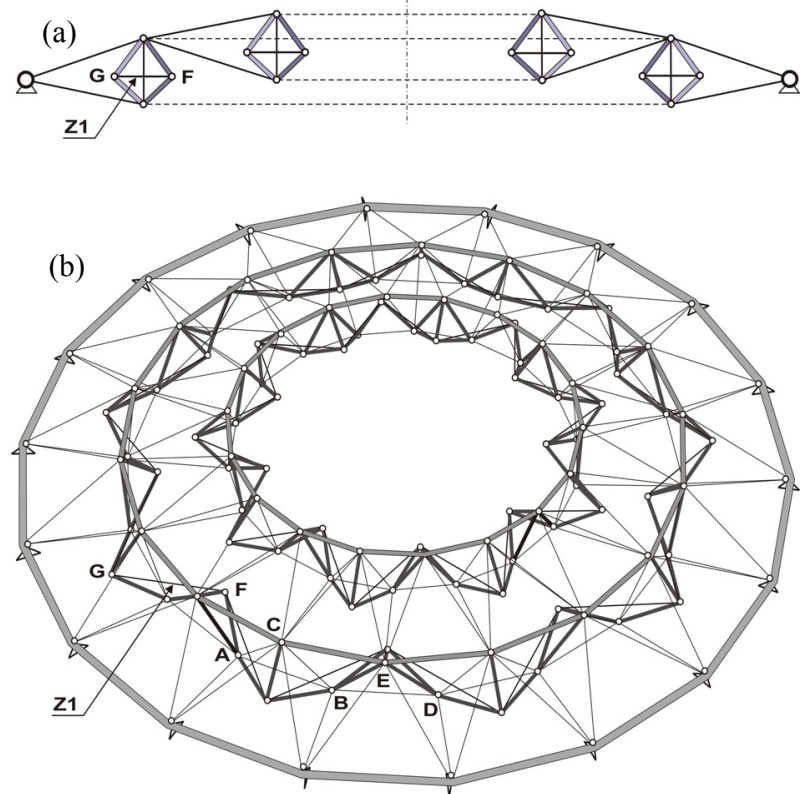

Fig. 10 Example of structural system consisting of single spatial hoops shaped by means of alternately located tetrahedron modules: (a) scheme of the main vertical cross-section of a dome structure; (b) general view of an exemplary form of a dome structure designed by means of the VL-TensZ1dO tension structure. 

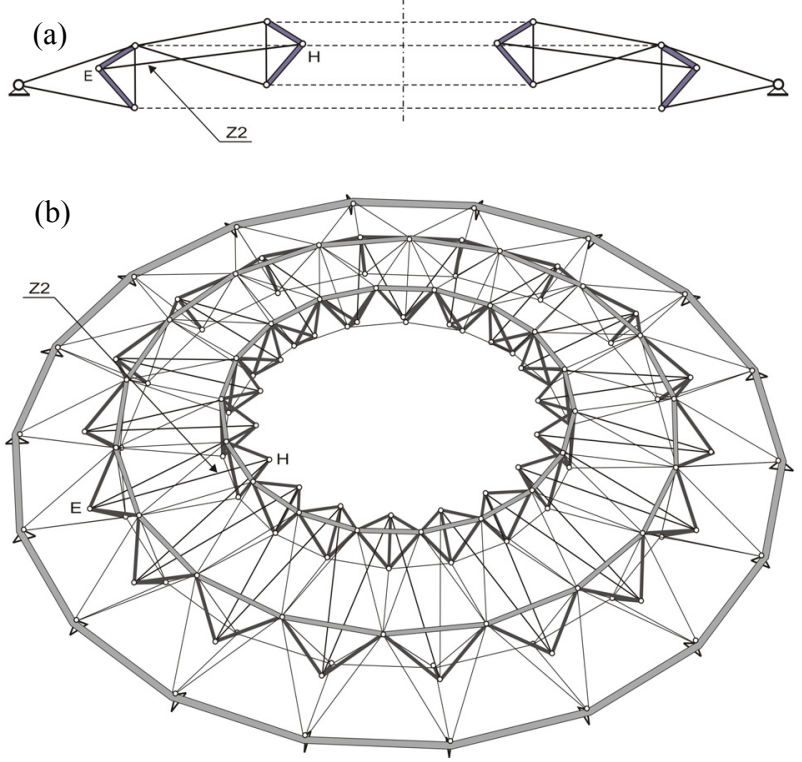

Fig. 11 (a) Scheme of the main vertical cross-section of a dome structure; (b) general view of the VUU-TensZ2O tension-strut structure designed for needs of a dome cover created by one double spatial hoop and shaped by tetrahedron modules spaced over upper triangular fields, where vertex nodes of modules are connected by tension members running in a zigzag way.
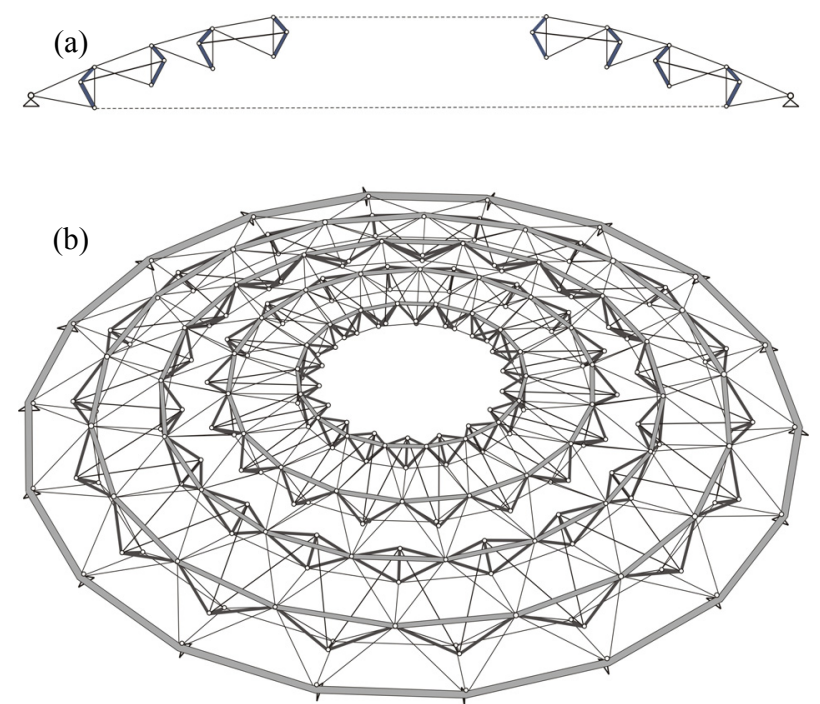

Fig. 12 Example of structural system built by means of two double spatial hoops: (a) scheme of vertical cross-section of a dome structure; (b) view of a complex form of the dome cover designed by means of the $2 x$ VUU-TensZ2O structure.

cross braces of each concentric hoop. Location of these struts is indicated by positions of struts BE and ED (as shown in Fig. 10b). These struts have to be arranged along selected edges of the tetrahedron modules, vertices of which are directed towards the dome center.

Similar recommendations and remarks refer to numerous group of the structural systems shaped by this type of tetrahedron modules spaced over the upper triangular fields of the hoop. Moreover, a special subgroup of these systems is built by means of these modules arranged in the mentioned fields along each of the two adjacent hoops. The two separate hoops have to be suitably connected together, due to which they can create a complex double hoop, as shown in Fig. 11. In this case, these modules are arranged over every second upper triangular field, but in each hoop they are directed oppositely. In the hoop nearest to the perimeter ring their vertices are directed towards the ring while in the successive hoops vertices of these modules are directed towards the dome centre.

Appropriate vertex nodes, for instance, nodes $\mathrm{H}$ and E (as shown in Fig. 11), are joined by means of tension members ( $\mathrm{Z} 2$ in Fig. 11) creating a specific zigzag configuration. The double form of the concentric hoop after the application of suitable pre-stressing has its own integral stiffness and supplemented by suitably designed boundary area it is denoted as the VUU-TensZ2O tension-strut structure. A large dome cover can be built by means of a greater number of such double hoops, while the intermediate parts between them have to be designed exactly in the shape of the boundary area of the previous structure. The structural configuration composed of two huge double hoops, shown in Fig. 12, is called the $2 x$ VUU-TensZ2O structure [18]. Initial structural analyses of all the presented spatial structures were done on bases of suitable numerical models defined in the programming language Formian [20].

\section{Conclusions}

All the presented systems were shaped from the results of structural transformations of a spherical form of the crystal space frame called a sunflower 
dome. One can expect that the structural formula of this dome is of a big develop potential and after further transformations, it can be used for defining, for example, a new system of retractable roof cover. The proposed tension-strut structures have to be suitably pre-stressed, so they can be applied as the lightweight support systems of the large span domes. Each of these structures has to be subjected to numerous comprehensive analyses carried out in order to estimate values of the necessary pre-stressing, sizes of the cross-sections of all component parts and other static and dynamic data. The variety of types of the invented systems enriches the scope of structural forms possible to apply in the design of the large span dome covers, which can obtain interesting and unique architectonic forms.

\section{References}

[1] F. Otto, Tensile Structures, The M.I.T. Press, Cambridge, Massachusetts, 1967.

[2] H. Berger, Light Structures - Structures of Light: The Art and Engineering of Tensile Architecture, Birkhäuser Verlag, Basel, Switzerland, 1996.

[3] E. Allen, W. Zalewski, Boston Structures Group, Form and Forces: Designing Efficient, Expressive Structures, John Wiley \& Sons, Inc., Hoboken, New Jersey, 2010.

[4] A. Hanaor, Developments in tensegrity systems: An overview, Space Structures 4 (2) (1993) 987-997.

[5] R. Motro, Tensegrity: Structural Systems for the Future, Kogan Page Science, London, 2003.

[6] B.B. Wang, A study on large span applications of non-contiguous strut tensegrity grids, in: International Symposium on Theory, Design and Realization of Shell and Spatial Structures, Japan, 2001.

[7] M. Levy, Limit of spatial structures, in: Proceedings of the Asia-Pacific Conference on Shell and Spatial
Structures, Beijing, 1996, pp. 3-9.

[8] D.H. Geiger, History of the Air Structure, Takenaka Komuten C., Ltd., Japan, 1988, pp. 4-23.

[9] J. Schlaich, R. Bergermann, Leicht Weit, Light Structures, Prestel, New York, 2003.

[10] M. Mengeringhausen, Space structures as means of individual shaping of buildings, in: Proceedings of the Third International Conference on Space Structures, London, 1984, pp. 73-80.

[11] Z.S. Makowski, Space Structures-A Review of the Developments within the Last Decade, Space Structures 4, Thomas Telford, London, 1993, pp. 1-8.

[12] M. Kawaguchi, Engineering aspects of space frames, in: Proceedings of the International Association for Shell and Spatial Structures Colloquium, Madrid, 1997, pp. 51-62.

[13] J. Rebielak, Space structures-Proposals for shaping, International Journal of Space Structures 7 (3) (1992) 175-190.

[14] D.H. Geiger, A. Stefaniuk, D. Chen, The design and construction of two cable domes for the Korean Olympics, in: Proceedings of the IASS Symposium on Shells, Membranes and Space Frames, Osaka, 1986, pp. 265-272.

[15] D.H. Geiger, Roof structure, US Patent, 4736553 A (1988).

[16] T. Robbin, Engineering a New Architecture, Yale University Press, New Haven, 1996.

[17] J. Rebielak, Structural systems of cable domes composed of concentric spatial hoops, in: International Symposium on Theory, Design and Realization of Shell and Spatial Structure, Nagoya, 2001, pp. 328-329.

[18] J. Rebielak, Shaping of Space Structures: Examples of Applications of Formian in Design of Tension-Strut Systems, Publishing House of the Wroclaw University of Technology, Wrocław, 2005.

[19] Z. Bać, J. Rebielak, Author's design study of solution of the Expo 2010 central complex, Architectus 1 (15) (2004) 83-89.

[20] H. Nooshin, P. Disney, Formex configuration processing I, International Journal of Space Structures 15 (1) (2000) $1-52$. 